\title{
MEDICATION USE FOLLOWING BARIATRIC SURGERY: FACTORS ASSOCIATED WITH EARLY DISCONTINUATION
}

\author{
Amy L. Kennedy, M.P.H. ${ }^{a, b}$, Tracy Nelson, Ph.D. ${ }^{a, b}$, Stefan Pettine, M.D.c ${ }^{c}$ Benjamin F. \\ Miller, Ph.D. ${ }^{a, b},{ }^{,}$, and Karyn L. Hamilton, Ph.D. ${ }^{a}{ }^{*}$ \\ aDepartment of Health and Exercise Science, Colorado State University, Fort Collins, CO 80523 \\ bColorado School of Public Health, Colorado State University, Fort Collins, CO 80523 \\ 'Northern Colorado Surgical Associates, Fort Collins, CO 80528
}

\begin{abstract}
Background-Medication discontinuation is a common result of bariatric surgery. The influence of individual patient characteristics and surgical outcomes on overall and specific medication discontinuation is not well understood. The purpose of the current study was to assess changes in medication use and identify individual characteristics and surgical outcomes associated with medication discontinuation among bariatric patients.

Methods-The patients included in the current study received bariatric surgery from the Northern Colorado Surgical Associates of Fort Collins, Colorado between October 2007 and September 2010. Demographic, weight, health, and medication data from 400 patients with at least one 6 or 12 month post-operative appointment were extracted from the Bariatric Outcome Longitudinal Database (BOLD). Multivariate regression analyses were used to investigate how patient factors affect total medication use over time, use of medications grouped by co-morbidity postoperatively, and use of specific medication classes post-operatively.
\end{abstract}

Results-Baseline co-morbidities, particularly type 2 diabetes (T2DM), male sex, and Roux-en$\mathrm{Y}$ gastric bypass surgery were significantly associated with decreased total medication use following surgery. Weight loss, systemic disease, sex, baseline co-morbidities, surgical complications, and race were significantly associated with continued use of specific medications following surgery.

Conclusions-Bariatric surgery can help patients with certain characteristics discontinue medications, but is not effective for all patients. Baseline health, sex, race, bariatric procedure, surgical complications, and post-operative weight loss may affect how bariatric patients' medication use changes pre-operatively to post-operatively.

Corresponding Author: Karyn L. Hamilton, PhD, Health and Exercise Science, Campus 1582, Colorado State University, Fort Collins, CO 80523-1582, Fax: (970) 491-0445, Karyn.hamilton@ colostate.edu.

Indicates Co-Principle Investigators

Conflict of Interest

Amy Kennedy reports no conflict of interest. Dr. Tracy Nelson reports no conflict of interest. Dr. Stefan Pettine reports no conflict of interest. Dr. Benjamin Miller reports no conflict of interest. Dr. Karyn Hamilton reports no conflict of interest. 


\section{Keywords}

bariatric surgery; medication; discontinuation; co-morbidity; obesity

\section{Introduction}

Obesity is associated with many co-morbidities and increased medication use[1-3]. Previous studies demonstrate decreased medication use following bariatric surgery[4, 5]. However, not all medications are equally likely to be discontinued. Patients tend to discontinue medications for type 2 diabetes (T2DM), hypertension, and hyperlipidemia more readily than medications for depression and hypothyroidism[5-7]. Medication discontinuation following bariatric surgery is a complex process involving patient characteristics, surgical outcomes, and decisions by physicians and patients, both medically appropriate and inappropriate[8]. While decisions made by physicians and patients are difficult to assess, data are available to analyze relationships between patient characteristics, surgical outcomes, and medication use patterns following bariatric surgery. These relationships illustrating how patient characteristics and surgical outcomes contribute to changes in medication use from pre-surgery to post-surgery are poorly understood. Recognizing the characteristics of patients likely to discontinue medications using statistical analyses controlling for related factors that could otherwise confound the associations under investigation could help physicians better advise prospective bariatric patients on risks and benefits of bariatric surgery and how to maximize positive results. The purpose of this study was to assess changes in medication use and identify patient characteristics and surgical outcomes associated with medication discontinuation among bariatric patients.

\section{Materials and Methods}

\section{Study Population \& Data Collection}

Bariatric patient data was retrospectively extracted from the Surgical Review Corporation's Bariatric Outcome Longitudinal Database (BOLD), which includes Northern Colorado Surgical Associates' (Fort Collins, CO) patients. Patients must have received bariatric surgery from the Northern Colorado Surgical Associates between October 2007 and September 2010 with at least one follow-up appointment occurring 4-15 months after surgery, as documented in BOLD. Using computer-generated, randomly assigned chart numbers, 400 de-identified patients were analyzed. Extracted data including demographics, health measures, and medication use were entered into a database (IBM SPSS Statistics version 20). Medication names extracted from BOLD were reclassified by drug class. Data from pre-operative, 4-8 month (6 months postoperative), and 9-15 months (12 months postoperative) appointments were included. Not all patients had an appointment at both the 6 and 12 month post-operative time points.

\section{Statistical Analyses}

Logistic regression was used to identify factors predictive of changes in: (1) total medication use over time for the entire study population and stratified by bariatric procedure (Roux-en$\mathrm{Y}$ gastric bypass and laparoscopic banding) by estimating models for the outcome of 
patients taking fewer total medications from the first time point to the second time point across four time periods; (2) medications for common co-morbidities following surgery by estimating models for the outcome of patients taking at least one drug from the medication classes within the co-morbidity group of interest at the patients' last endpoints (either 6 or 12 months post-operative depending on appointment attendance); and (3) use of the most common classes of medications (Table 1) postoperatively by estimating models for the outcome of patients taking at least one drug from these common classes of medications at the patients' last endpoints. Age, sex, race, payment method, pre-operative body mass index (BMI), bariatric procedure, American Society of Anesthesiologists (ASA) class, excess body weight (EBW) loss, surgical complication, and co-morbidities were individually regressed with each outcome. The medical reasons for medication use and discontinuation were unavailable in BOLD and therefore were not included in the analysis. Variables correlated with the outcome at $\mathrm{p} \unlhd 0.09$ were then entered into a multivariate, stepwise logistic regression model. Final multivariate models included variables associated with the outcome at $\mathrm{p} \unlhd .05$ when controlling for all other variables in the model. Pre-operative use of medications relevant to the outcome of interest was also controlled for in each model. Analyses were performed using SAS 9.3.

\section{Results}

\section{Total Medication Use Over Time}

Baseline T2DM was the most consistent positive predictor for decreased number of total medications across all time periods studied, with odds ratios ranging from 2.693 to 5.096 (Table 2). Baseline hypertension, GERD, and asthma were also positively associated with fewer medications (Table 2). Roux-en-Y gastric bypass patients were almost 2 times more likely than laparoscopic banding patients to discontinue medications between baseline and 6 months postoperative and baseline to their last endpoint (Table 2). Sex was also associated with changes in medication use patterns, with males almost 2.5 times more likely than females to take fewer medications from baseline to 12 months post-operative (Table 2).

\section{Use of Medications Grouped by Co-morbidity}

The analyses conducted to estimate associations between patient factors and continued use of medications grouped by co-morbidity revealed increasing age was associated with taking medications typically prescribed for hypertension, T2DM, pain, GERD, hyperlipidemia, and CVD (Table 3). Increasing EBW loss was associated with post-operative discontinuation of medications commonly prescribed for hypertension, asthma, and CVD (Table 3). Several baseline co-morbidities were associated with post-operative medication use by disease group (Table 3). Positive associations were significant for baseline T2DM and continued use of common hypertension medications, baseline depression and continued use of T2DM medications, and baseline sleep apnea and continued use of mental health medications (Table 3). No predictive factors analyzed were associated with gastrointestinal, urinary, reproductive, or infection medication use post-operatively. 


\section{Use of Medications from Specific Drug Classes}

Many statistically significant associations were discovered in the analyses of relationships between patient characteristics and continued use of specific classes of drugs (Table 4). Females were about 5 times more likely than males to continue with antiepileptic drugs (Table 4). Patients of Hispanic ethnicity were about 10 times as likely as Caucasian patients to continue taking Angiotensin converting enzyme inhibitors/Angiotensin II receptor blockers (ACE/ARBs) (Table 4). Losing more excess body weight following surgery was associated with discontinuing insulin, anithypertensives/diuretics, and beta blockers (Table 4). Several baseline co-morbidities were associated with continued use of medications from specific drug classes, including the presence of baseline sleep apnea associated with the continued use of antidepressants and baseline T2DM associated with the continued use of beta blockers (Table 4).

\section{Discussion}

The current study demonstrates medication discontinuation following bariatric surgery varies by patient characteristics and surgical outcomes. Baseline co-morbidities, particularly T2DM, weight loss, sex, race, and surgical compilations are important variables found to have significant relationships with medication use patterns among bariatric patients. To our knowledge, this is the first use of multivariate logistic regression to elucidate associations between patient factors and changes in medications commonly prescribed for hypertension, T2DM, pain, mental health, asthma, GERD, hyperlipidimia, and CVD.

The regression method used facilitates a better understanding of how patient factors and surgical outcomes influence medication discontinuation for a variety of common medications when controlling for factors such as age, sex, weight loss, baseline comorbidities, and baseline medication use. Controlling for a variety of individual factors, including those investigated in other analyses and some not previously explored, provides a greater level of certainty the reported associations between patient characteristics and medication discontinuation are valid and are not attributable to another variable. The multivariate regression method provides a greater level of certainty the reported relationships exist than other methods previously used to assess changes in medication associated with bariatric surgery because the multivariate regression method assures the magnitude and significance of reported relationships are independent from other important individual variables included in the model. (Reviewer \#1) By analyzing three levels of medication use, (1) total, (2) grouped by co-morbidity, and (3) specific drug classes, the results contribute to a more nuanced understanding of which patients discontinue medications and why. Using the multivariate regression method with 3 levels of analyses allows for a more specific evaluation of which variables are associated with changes in overall medication use as well as which variables are associated with changes in the use of specific drugs. (Reviewer \#1) While not all associations may be clinically important and association does not imply causation, highlighting the relationships between patient variables and medication use patterns is important for beginning to understand the complex nature of medication changes following bariatric surgery. 


\section{Total Medication Use}

Reduction in mean medication use-An overall $50 \%$ reduction in medication use occurred 6 months post-operatively and was sustained 12 months post-operative for this study's patient population (Table 5). Our results agree with previous studies reporting similar decreases in medication use following bariatric surgery $[4,9,10]$.

\section{Baseline co-morbidities were associated with overall medication}

discontinuation-Patients with baseline T2DM were about 3 times more likely than patients without T2DM to decrease the number of medications in use following surgery when controlling for other factors (Table 2). Patients with baseline hypertension, GERD, or asthma were also more likely to use fewer medications by 12 months post-operative, although discontinuation for patients with these co-morbidities were not as consistent as overall medication discontinuation for T2DM patients (Table 2). Therefore, overall medication discontinuation following bariatric surgery may be dependent on the specific comorbidities present before surgery, and patients with hypertension, GERD, asthma, and particularly T2DM may be more successful in reducing the number of medications in use following bariatric surgery. Although other studies have demonstrated rates of medication discontinuation differ by use [5-7, 10-12], to our knowledge this is the first documentation of a relationship between baseline co-morbidities and changes in overall medication use for bariatric patients.

Overall medication discontinuation varies by surgery type-Roux-en-Y gastric bypass patients were almost 2 times more likely than laparoscopic banding patients to decrease total medications post-operatively (Table 2 ). These results are consistent with previous comparisons of medication change in these surgery groups [13], although to our knowledge this is the first time the relationship has been documented when controlling for other factors such as weight loss, baseline co-morbidities, sex, and age. Among Roux-en-Y patients, males and those with baseline T2DM, hypertension, GERD, asthma, and alcohol use, were more likely to decrease medications by 12 months post-op (not shown). Among laparoscopic banding patients, baseline T2DM and lower extremity edema were associated with decreased medications (not shown). Medication discontinuation therefore varies by surgery type among all patients and varies by sex and co-morbidities within gastric bypass and laparoscopic banding groups. Although each association may not be clinically relevant, patients considering bariatric surgery and their physicians could potentially make more informed decisions about the most appropriate type of surgery by understanding relationships between baseline patient characteristics, different types of surgery, and postoperative medication discontinuation.

\section{Grouped and Specific Medication Use}

Rates of medication discontinuation vary by medication type-Medications typically prescribed for hypertension, GERD, hyperlipidemia, CVD, and T2DM were most often discontinued following bariatric surgery, whereas medications commonly used for hypothyroidism, mental health, pain, and asthma were less likely to be discontinued (Table 1). Similarly, previous studies have found more pronounced discontinuation of medications for CVD, T2DM, gastroenterological issues, hyperlipidemia, and arthritis, and less 
pronounced discontinuation for infection, pain, respiratory, psychotropic, depression, and thyroid medications [5-7, 10, 12].

\section{Weight loss is associated with discontinuation of specific medications-}

Weight loss was associated with discontinuing hypertension, asthma, and CVD medications following bariatric surgery (Table 3). More specifically, weight loss was associated with discontinuing insulin, antihypertensive/diuretics, and beta blockers following surgery (Table 4). These results indicate the association between weight loss and discontinuation of hypertension and CVD medications may be mediated by discontinuation of antihypertensives/diuretics and beta blockers. Therefore, patients able to lose more weight following bariatric surgery may experience more pronounced decreases in asthma medications, insulin, antihypertensives/diuretics, and beta blockers following bariatric surgery.

\section{ASA class, sex, complications, and race are associated with specific} medication discontinuation-For every increase in ASA class, patients were about 2 times more likely to continue using T2DM and mental health medications (Table 3). Therefore, more severe systemic disease at baseline may predict continued use of medications for T2DM and mental health disorders postoperatively. Within our defined medication groups, sex was only significantly associated with continued mental health medication use. Females were about 4 times more likely to continue taking mental health medications post-operatively (Table 3 ), an association that appears to be mediated by a strong, positive association between females and continued use of antiepileptic medications (Table 4). The medical reason for antiepileptic medication use was unavailable for this analysis, but antiepileptic medications are used more often for psychiatric and pain disorders than seizure disorders in the general population[14]. If this trend holds true in the bariatric patient population, these results may indicate females with psychiatric or pain disorders are more likely to continue with antiepileptic medications than males with these disorders.

Interestingly, patients experiencing surgical complications were about 4 times more likely to continue GERD medications post-operatively (Table 3). Certain surgical complications, such as band slippage $[15,16]$, have been indicated in increasing the risk of GERD following surgery. Additionally, patients with GERD prior to surgery may be more likely to experience surgical complications than patients with other obesity-related comorbidities[17]. The relationship between surgical complications and GERD medication use may therefore be bidirectional.

Although the sample population was overwhelmingly Caucasian (Table 5), we found a strong association between Hispanic race and post-operative ACE/ARB use (Table 4). Because the analysis controlled for common co-morbidities, baseline medication use, and other pertinent variables that could confound this association, further investigation into unaccounted for social or behavioral mediators of this relationship may be warranted.

\section{Baseline co-morbidities are associated with specific medication use-Our} results revealed intriguing relationships between baseline co-morbidities and the use of specific medications. Baseline T2DM was associated with continued use of hypertension 
medications (Table 3 ), which was likely mediated by the continued use of beta blockers postoperatively (Table 4). This is consistent with findings that patients taking beta-blockers have a higher risk of developing T2DM[18]. Baseline depression was associated with continued use of T2DM medications (Table 3). Similarly, others have found depressed individuals are more likely to develop T2DM[19]. Baseline sleep apnea was associated with continued use of mental health medications (Table 3), a relationship likely mediated by the continued use of antidepressants among patients with baseline sleep apnea (Table 4). These results are consistent with previously documented links between sleep apnea and depression[20, 21].

\section{Limitations}

The time period studied of 6 to 12 months post-operative limits any conclusions about longterm changes in medications following bariatric surgery. We also did not have both 6 and 12 month post-operative data for every patient due to noncompliance and our inclusion of patients receiving surgery less than 9 months before data extraction. However, the shorter time period and less restrictive inclusion criteria facilitated use of a larger and more representative cross-section of bariatric patients. Our sample population was primarily female and Caucasian; homogeneity likely limited our ability to detect differences in medication use between groups and limits our ability to generalize our findings to more diverse bariatric populations. However, we still detected associations between sex, race, and medication use. We did not know for certain that medication classes grouped under specific co-morbidities were prescribed to patients for that co-morbidity. To address this, we analyzed specific medication classes to parse out individual relationships between patient factors and specific medications irrespective of reason for prescription. Additionally, we were unable to analyze whether medication discontinuation was medically appropriate or inappropriate. We therefore can only analyze medication use patterns and cannot make firm conclusions about changes in co-morbidity severity. Finally, we had no access to behavioral and social variables that could help elucidate relationships between bariatric patient factors and medication use changes.

\section{Conclusions}

Although medication discontinuation is only one possible favorable outcome following successful bariatric surgery, taking less medication can significantly enhance patients' quality of life. Our results demonstrate bariatric surgery can help patients with certain characteristics discontinue medications within a year following surgery, but is not effective for medication discontinuation among all patients. Baseline co-morbidities, particularly T2DM, male sex, and Roux-en-Y surgery were associated with decreased total medication use following surgery when controlling for other variables of interest. Weight loss, systemic disease, sex, baseline co-morbidities, surgical complications, and race were associated with specific medication use following surgery when controlling for other variables of interest. Determining which patients are more likely to benefit from bariatric surgery by understanding the relationships between individual factors and changes in medication use may help physicians better advise patients considering bariatric surgery. 


\section{Acknowledgments}

Thank you to Dr. Elise Donovan for technical assistance and Becki Mudgett for patient coordination. The Colorado School of Public Health provided graduate research assistant funding.

\section{References}

1. Must A, Spadano J, Coakley EH, et al. The disease burden associated with overweight and obesity. JAMA. 1999; 282:1523-9. [PubMed: 10546691]

2. Kopelman P. Health risks associated with overweight and obesity. Obes Rev. 2007; 8 (Suppl 1):137. [PubMed: 17316295]

3. Sturm R. The effects of obesity, smoking, and drinking on medical problems and costs. Health Aff (Millwood). 2002; 21:245-53. [PubMed: 11900166]

4. Dunkle-Blatter SE, St Jean MR, Whitehead C, et al. Outcomes among elderly bariatric patients at a high-volume center. Surg Obes Relat Dis. 2007; 3:163-9. discussion 9-70. [PubMed: 17331804]

5. Cremieux PY, Ledoux S, Clerici C, et al. The impact of bariatric surgery on comorbidities and medication use among obese patients. Obes Surg. 2010; 20:861-70. [PubMed: 20440579]

6. Malone M, Alger-Mayer SA. Medication use patterns after gastric bypass surgery for weight management. Ann Pharmacother. 2005; 39:637-42. [PubMed: 15741419]

7. Segal JB, Clark JM, Shore AD, et al. Prompt reduction in use of medications for comorbid conditions after bariatric surgery. Obes Surg. 2009; 19:1646-56. [PubMed: 19763709]

8. Mechanick JI, Kushner RF, Sugerman HJ, et al. American Association of Clinical Endocrinologists, The Obesity Society, and American Society for Metabolic \& Bariatric Surgery medical guidelines for clinical practice for the perioperative nutritional, metabolic, and nonsurgical support of the bariatric surgery patient. Obesity (Silver Spring). 2009; 17 (Suppl 1):S1-70. v. [PubMed: 19319140]

9. Nelson LG, Lopez PP, Haines K, et al. Outcomes of bariatric surgery in patients $>$ or $=65$ years. Surg Obes Relat Dis. 2006; 2:384-8. [PubMed: 16925358]

10. Snow LL, Weinstein LS, Hannon JK, et al. The effect of Roux-en-Y gastric bypass on prescription drug costs. Obes Surg. 2004; 14:1031-5. [PubMed: 15479590]

11. Ahroni JH, Montgomery KF, Watkins BM. Laparoscopic adjustable gastric banding: weight loss, co-morbidities, medication usage and quality of life at one year. Obes Surg. 2005; 15:641-7. [PubMed: 15946454]

12. Cunningham JL, Merrell CC, Sarr M, et al. Investigation of antidepressant medication usage after bariatric surgery. Obes Surg. 2012; 22:530-5. [PubMed: 21901283]

13. O'Keefe KL, Kemmeter PR, Kemmeter KD. Bariatric surgery outcomes in patients aged 65 years and older at an American Society for Metabolic and Bariatric Surgery Center of Excellence. Obes Surg. 2010; 20:1199-205. [PubMed: 20532834]

14. Kaufman KR. Antiepileptic drugs in the treatment of psychiatric disorders. Epilepsy Behav. 2011; 21:1-11. [PubMed: 21498130]

15. Lee CW, Kelly JJ, Wassef WY. Complications of bariatric surgery. Curr Opin Gastroenterol. 2007; 23:636-43. [PubMed: 17906440]

16. de Jong JR, van Ramshorst B, Timmer R, et al. Effect of laparoscopic gastric banding on esophageal motility. Obes Surg. 2006; 16:52-8. [PubMed: 16417759]

17. Cawley J, Sweeney MJ, Kurian M, et al. Predicting complications after bariatric surgery using obesity-related co-morbidities. Obes Surg. 2007; 17:1451-6. [PubMed: 18219771]

18. Gress TW, Nieto FJ, Shahar E, et al. Hypertension and antihypertensive therapy as risk factors for type 2 diabetes mellitus. Atherosclerosis Risk in Communities Study. N Engl J Med. 2000; 342:905-12. [PubMed: 10738048]

19. Knol MJ, Twisk JW, Beekman AT, et al. Depression as a risk factor for the onset of type 2 diabetes mellitus. A meta-analysis. Diabetologia. 2006; 49:837-45. [PubMed: 16520921]

20. Qureshi A, Ballard RD. Obstructive sleep apnea. J Allergy Clin Immunol. 2003; 112:643-51. quiz 52. [PubMed: 14564340]

Obes Surg. Author manuscript; available in PMC 2015 May 01. 
21. Aloia MS, Arnedt JT, Smith L, et al. Examining the construct of depression in obstructive sleep apnea syndrome. Sleep Med. 2005; 6:115-21. [PubMed: 15716215] 


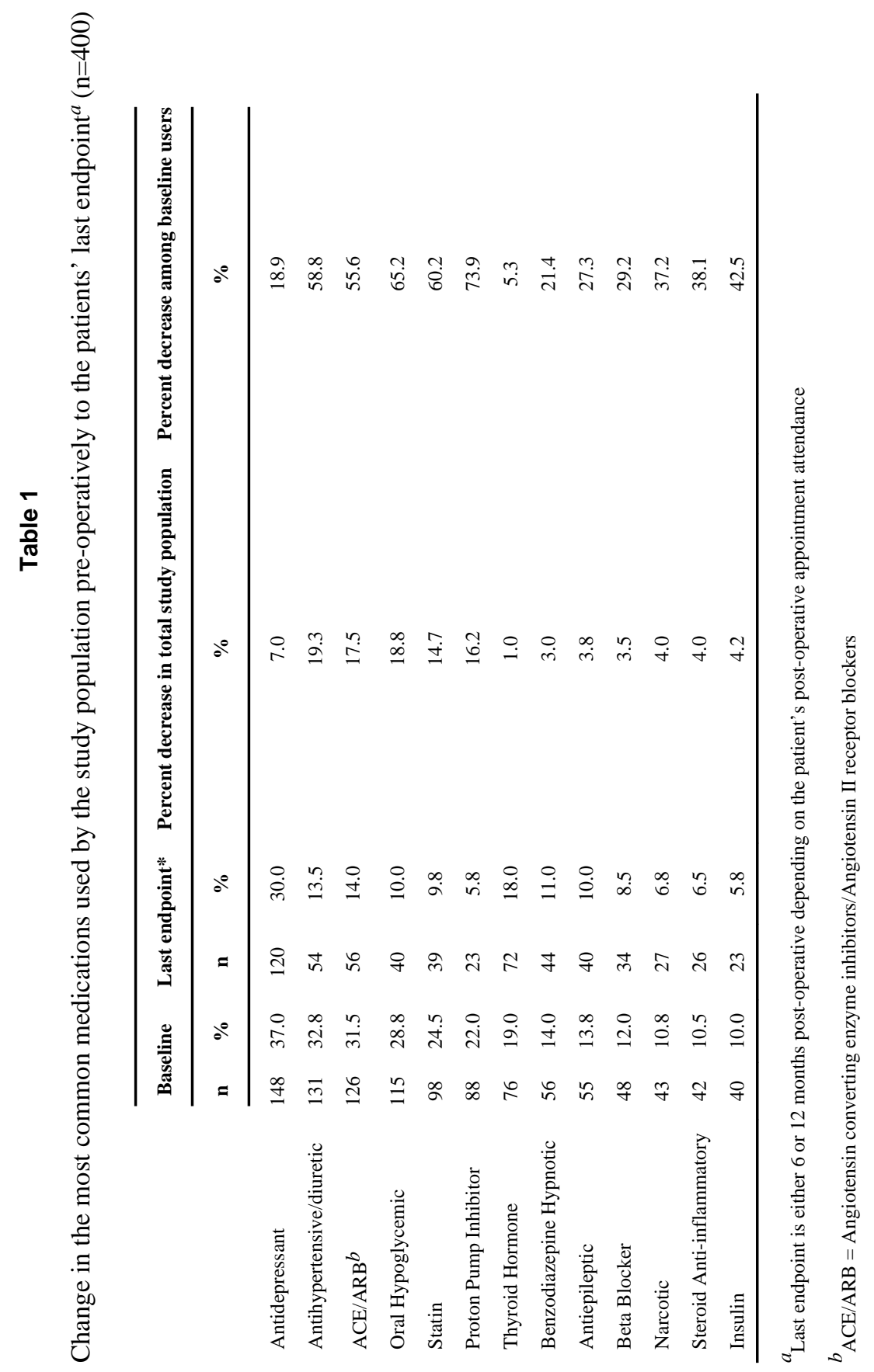

Obes Surg. Author manuscript; available in PMC 2015 May 01. 
Table 2

Variables associated with decreased total medication use over time for four time periods

\begin{tabular}{|c|c|c|c|c|}
\hline Variable & $\begin{array}{l}\text { Baseline to } 6 \text { mo. } \\
\qquad(\mathrm{n}=353)\end{array}$ & $\begin{array}{l}\text { Baseline to } 12 \text { mo. } \\
\qquad(n=277)\end{array}$ & $\begin{array}{l}6 \text { months to } 12 \text { mo. } \\
(n=230)\end{array}$ & $\begin{array}{c}\text { Baseline to last } \\
\text { endpoint }{ }^{c}(\mathrm{n}=400)\end{array}$ \\
\hline Age (continuous) & $1.024(1.001-1.047)$ & - & $1.031(1.001-1.062)$ & - \\
\hline Male (v. female) & - & $2.427(1.078-5.464)$ & - & - \\
\hline $\begin{array}{l}\text { Roux-en-Y gastric bypass } \\
\text { (v. laparoscopic banding) }\end{array}$ & $1.978(1.203-3.253)$ & - & - & $1.738(1.096-2.755)$ \\
\hline Baseline hypertension & $1.970(1.167-3.327)$ & - & - & $2.432(1.545-3.829)$ \\
\hline Baseline type 2 diabetes & $2.693(1.635-4.438)$ & $5.096(2.780-9.339)$ & $3.064(1.541-6.093)$ & $3.226(1.996-5.216)$ \\
\hline Baseline GERD $^{d}$ & $1.960(1.223-3.143)$ & $2.388(1.370-4.161)$ & - & $2.236(1.431-3.494)$ \\
\hline Baseline asthma & - & $4.154(1.336-12.913)$ & $2.797(1.104-7.086)$ & - \\
\hline
\end{tabular}

${ }^{a}$ Data presented as odds ratio (95\% confidence interval)

${ }^{b}$ - indicates no association at $\mathrm{p} \unlhd 0.05$

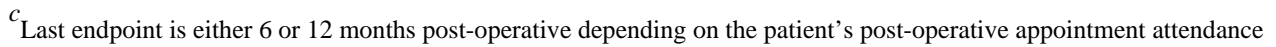

$d_{\text {GERD }}=$ Gastroesophageal reflux disease 
Table 3

Variables associated with use of medications grouped by disease at patients' last endpoint ${ }^{a}$

\begin{tabular}{|c|c|c|c|c|c|c|c|c|}
\hline \multirow[b]{2}{*}{ Variable } & \multicolumn{8}{|c|}{ Medications use to treat } \\
\hline & Hypertensiong $g$ & Type 2 Diabetes $h$ & Pain $i$ & Mental Health $j$ & Asthma $k$ & GERD $l$ & Hyperlipidemia $\boldsymbol{m}$ & $\mathrm{CVD}^{n}$ \\
\hline Age (continuous) & $1.064(1.036-1.093)$ & $1.035(1.002-1.070)$ & $1.037(1.002-1.074)$ & - & - & $1.050(1.006-1.096)$ & $1.050(1.011-1.089)$ & $1.068(1.037-1.101)$ \\
\hline Female (v. male) & - & - & - & $4.254(1.673-10.816)$ & - & - & - & - \\
\hline Baseline BMI (categories) $d$ & $1.928(1.12-3.314)$ & - & - & - & - & - & - & $2.070(1.136-3.771)$ \\
\hline ASA Class $e$ & - & $2.035(1.001-4.138)$ & - & $2.004(1.060-3.789)$ & - & - & - & - \\
\hline $\begin{array}{l}\text { \% Excess body weight loss } \\
\text { (continuous) }\end{array}$ & $0.983(0.969-0.998)$ & - & - & - & $0.974(0.951-0.997)$ & - & - & $0.982(0.966-0.998)$ \\
\hline Complication $f$ & - & - & - & - & - & $4.108(1.492-11.312)$ & - & - \\
\hline Baseline type 2 diabetes & $1.995(1.155-3.445)$ & N/A & - & - & - & - & - & - \\
\hline Baseline depression & - & $\begin{array}{c}2.244(1.073- \\
4.693)\end{array}$ & - & N/A & - & - & - & - \\
\hline Baseline sleep apnea & - & - & - & $3.257(1.672-6.343)$ & - & - & - & - \\
\hline $\begin{array}{l}\text { Baseline lower extremity } \\
\text { edema }\end{array}$ & - & - & - & - & $4.305(1.620-11.436)$ & - & - & - \\
\hline Baseline urinary incontinence & - & - & - & - & $2.734(1.062-7.041)$ & - & - & - \\
\hline
\end{tabular}

${ }^{a}$ Last endpoint is either 6 or 12 months post-operative depending on the patient's post-operative appointment attendance

$b$

Data presented as odds ratio (95\% confidence interval)

$c$ - indicates no association at $\mathrm{p} \unlhd 0.05$

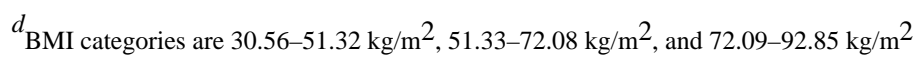

${ }^{e} \mathrm{ASA}=$ American Society of Anesthesiologists

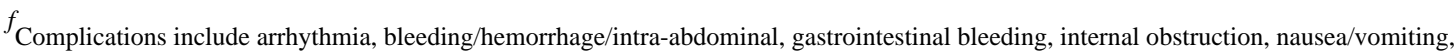
cholecystitis, internal hernia, adjustable gastric band slippage, and stricture

${ }^{g}$ Medication classes include angiotensin converting enzyme (ACE) inhibitors/angiotensin II receptor-blockers (ARB), antihypertensives/diuretics, alpha-blockers, beta-blockers, and calcium-blockers

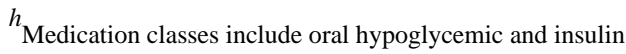

${ }^{i}$ Medication classes include narcotics, non-narcotic analgesics, muscle relaxants, and non-steroidal anti-inflammatory

${ }^{j}$ Medication classes include antidepressants, anti-psychotics, anti-anxiety agents, anti-epileptics, sedatives, stimulants, and benzodiazepine hypnotics

${ }^{k}$ Medication classes include leukotriene inhibitors, steroid anti-inflammatory, antihistamines, and bronchodilators

${ }^{l}$ Medication classes include histamine-2-blockers and proton pump inhibitors

${ }^{m}$ Medication classes include statins, lipid reducers, and niacin

${ }^{n}$ Medication classes include antiarrhythmics, anticoagulants, beta blockers, calcium blocker, ACE/ARB, nitrates, and vasodilators

${ }^{o}$ Models for each medication group control for baseline use of medication classes within that group 


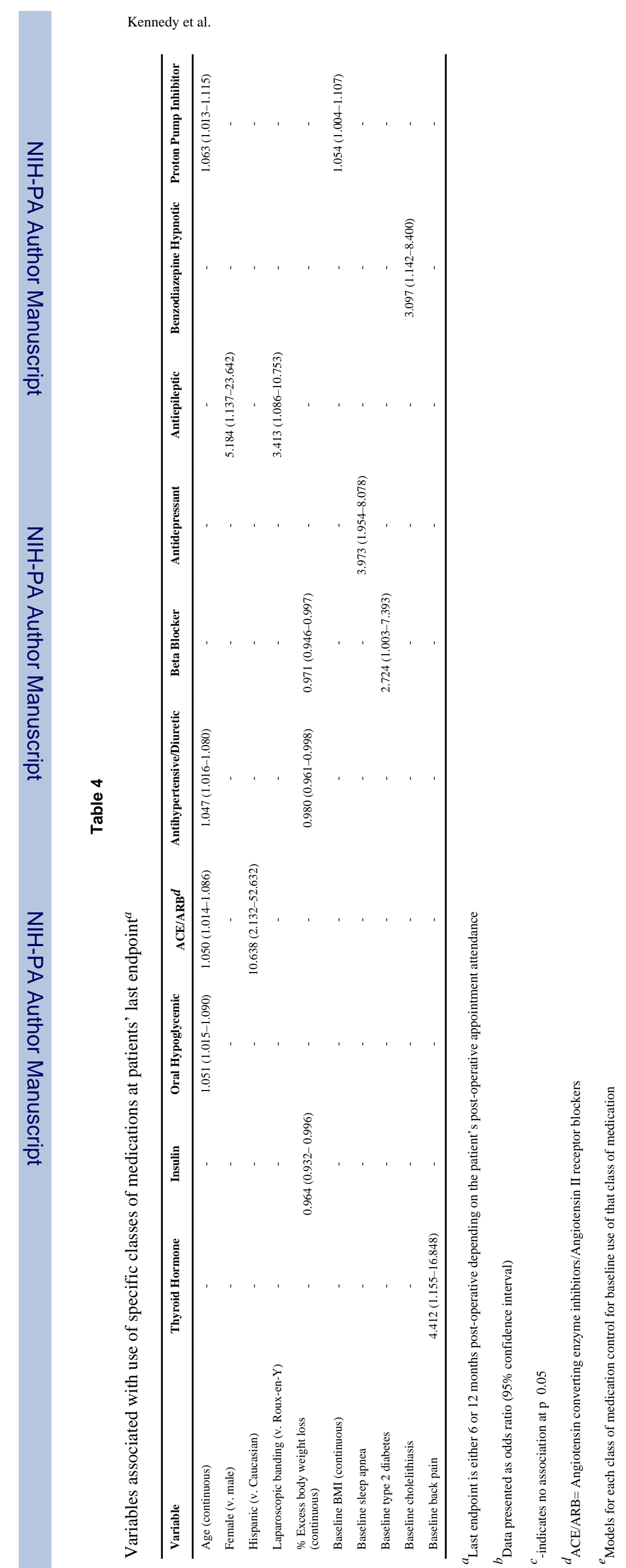

Page 13 


\section{Table 5}

Characteristics of the study population $(n=400)$

\begin{tabular}{|c|c|}
\hline Characteristic & Percent \\
\hline \multicolumn{2}{|l|}{ Sex } \\
\hline Female & 81 \\
\hline Male & 19 \\
\hline \multicolumn{2}{|l|}{ Race } \\
\hline Caucasian & 96 \\
\hline Hispanic & 4 \\
\hline \multicolumn{2}{|l|}{ Payment Method } \\
\hline Private Insurance & 60 \\
\hline Medicaid & 22 \\
\hline Medicare & 10 \\
\hline Other & 9 \\
\hline \multicolumn{2}{|l|}{ Bariatric procedure } \\
\hline Roux-en-Y gastric bypass & 66 \\
\hline Laparoscopic banding & 34 \\
\hline \multicolumn{2}{|l|}{ ASA Class ${ }^{a}$} \\
\hline Status $2^{b}$ & 6 \\
\hline Status $3^{c}$ & 68 \\
\hline Status $4^{d}$ & 26 \\
\hline Surgical complication & 17 \\
\hline Pre-discharge complication & 7 \\
\hline Post-discharge complication & 11 \\
\hline \multicolumn{2}{|l|}{ Follow-up appointment attendance } \\
\hline 6 months & 88 \\
\hline 12 months & 69 \\
\hline
\end{tabular}

\begin{tabular}{lcc}
\cline { 2 - 3 } & Mean \pm SD & Range \\
\cline { 2 - 3 } Age $(\mathrm{yrs})$ & $48 \pm 12$ & $18-78$ \\
Weight $(\mathrm{lbs})$ & & \\
Baseline & $291.0 \pm 63.3$ & $175.0-545.0$ \\
6 months post-operative & $227.7 \pm 51.2$ & $139.0-502.0$ \\
12 months post-operative & $208.6 \pm 52.5$ & $114.0-509.0$ \\
BMI (kg/m $\left.{ }^{2}\right)^{e}$ & & \\
Baseline & $46.99 \pm 8.68$ & $30.56-92.85$ \\
6 months post-operative & $36.81 \pm 7.44$ & $20.97-86.18$ \\
12 months post-operative & $33.86 \pm 7.46$ & $22.31-87.44$ \\
EBW (lbs) $f$ & & \\
Baseline & & \\
6 months post-operative & $94.8 \pm 46.0$ & $0.0-379.0$
\end{tabular}

Obes Surg. Author manuscript; available in PMC 2015 May 01. 
12 months post-operative

EBW loss from

Baseline (\%)

6 months post-operative

12 months post-operative

Medications in Use

Baseline $(n=400)$

6 months post-operative $(\mathrm{n}=353)$

12 months post-operative $(\mathrm{n}=277)$

${ }^{a} \mathrm{ASA}=$ American Society of Anesthesiologists

${ }^{b}$ ASA Class $2=$ mild systemic disease

${ }^{c}$ ASA Class $3=$ severe systemic disease

$d_{\text {ASA Class } 4}=$ severe systemic disease that is a constant threat to life

$e_{\mathrm{BMI}}=$ Body Mass Index

$f_{\text {EBW=Excess Body Weight }}$

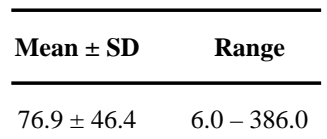

$41.0 \pm 15.5 \quad 0.0-100.0$

$51.4 \pm 20.4 \quad 0.0-94.5$

$4.0 \pm 3.4 \quad 0-17$

$2.3 \pm 2.5 \quad 0-12$

$2.1 \pm 2.4 \quad 0-13$ 